\title{
LI-FI: An Emerging Technology for Health Care
}

\author{
M. Pandiyan ${ }^{1}$ and C. S. Revathi ${ }^{2}$ \\ ${ }^{1 \& 2}$ Assistant Professor \\ ${ }^{1}$ Department of Computer Science, Mahendra Engineering College, Tamil Nadu, India \\ ${ }^{2}$ Department of Commerce, Sri Vasavi College, Tamil Nadu, India \\ E-Mail: pandiyanm@mahendra.info,revathisudarsan@gmail.com
}

\begin{abstract}
In today's world, everything around us are about to expose the harmful radiations. In India, there is no proper automatic monitoring system for the patients in the hospital. Even in the hospitals, the surgeries are done using Internet connectivity where WI-FI leads the way. The Wi-Fi technology uses RF spectrum which exhibits the harmful radiations. The LI-FI Technology will overcome this effect as it uses visible light as a source which is non-hazardous. Some of the death in the medical field happens due to the lack of proper electric facilities and monitoring system. By means of using LI-FI we can overcome the problems of power insufficiency and also the data transmission. Our Li-Fi kit will act as a mediator that converts any kind of visible light source into data for transmission. So, the one who has our kit can send and receive data in high speed even at a rate of several Gbps.

Keywords: Harmful Radiations, LI-FI, Non-Hazardous, Data

Transmission, Visible Light, Monitoring System.
\end{abstract}

\section{INTRODUCTION}

Now days, the availability of internet is very essential for all sort of works. Due to some signal problems we fail to access internet in some areas. In medical field the technology is improving day by day and still in India we are lagging in advanced technologies and patient monitoring in hospitals. The recent news about deaths of infants kept in incubator and also the deaths happening in the hospitals due the lack of monitoring. And also, in robotic surgeries the usage of Wi-Fi for internet connectivity may create hazards to patients due to the flow of radio waves. For a long time, medical technology has lagged behind the rest. The scope for wireless communication in the medical field is set on the rise; there are many devices which work on Wi-Fi such as infusion pumps, defibrillators, lung ventilators, and anesthesia machine. When a doctor is supposed to use magnetic resonance imaging scanners along with infusion pumps, which work on Wi- Fi there results a frequencyoverlapping problem.

The advancement of wireless devices, radio frequency (RF) spectrum increases regularly and accordingly it affects human body by diseases such as cancer, neurological disease, reproductive disorders, immune dysfunction, and electromagnetic hypersensitivity etc. This can be eliminated by Light fidelity (Li-Fi) one of the solution for the highspeed data network, proposed by a German physicist Harold Haas. Li-Fi networks support the transmission of data through illumination of light emitting diode (LED) bulb, thereby it is also termed as visible light communications (VLC). Li-Fi to free space optics as it also utilizes light to transfer data but it is difficult to lay optical fibres in hospitals. Parallel working with various EMI devices is feasible with $\mathrm{Li}-\mathrm{Fi}$ and is also beneficial for robotic surgeries and automated procedures. During surgery, Li-Fi system along with various sensors is needed to get immediate guidance from experts in the therapy by sharing data, videos/live details about the patient for the best results. With the help of Li-Fi technology we can enables clinicians to monitor patients remotely and give them timely health information, reminders, and support.

\section{NEED FOR THE PRODUCT}

At present in India there is no automatic monitoring equipment to update the minute by minute status of the patient. Especially in the case of robotic surgery, with the help of Li-Fi the robots can perform the complicated surgeries without any radiation and hazards to the patients since it emits only light emitting diodes. $\mathrm{Li}-\mathrm{Fi}$ is an alternate to Wi-Fi. The main disadvantage of Wi-Fi which lifi overcomes is, Wi-Fi uses RF spectrum which transfer the data at a specific speed whereas in lifi once the visible spectrum is used it is too fast than that of Wi-Fi. In Li Fi anyone who as access to the light can use the internet and it allows the users to move from one light source to another without any interruption. Table 1 shows the comparison between eh $\mathrm{Li}-\mathrm{Fi}$ and $\mathrm{Wi}-\mathrm{Fi}$ with respect to various parameters.

\section{METHODOLOGY}

Our product senses the environment through the different sensors and updates it to the doctors. Our Li-Fi product uses common household LED (light emitting diodes) light bulbs to enable data transfer, boasting speeds of up to 224 gigabits per second. Li-Fi signals are confined to narrowly-focused 'beams' that don't travel through walls. Moreover, LED lights are natural beam-formers, which make it easier to create separate uplink and downlink channels, which essentially mean more secure internet browsing. As we know safety is very important concern in hospitals. All the hospitals which use robotic equipment have and need secure environment for surgeries will need this product. If a doctor wants to consult from other expertise for anytreatment of patient then they can make a video conference in 
hospitals/OTs, so it will not affect patient's body and they can get best results. Generally, in ICUs nurses are taking care of abnormalities and the health of the patient but they may not be available for taking care of patient's health throughout the day with them hence we have proposed an automatic LI-FI based advanced patient monitoring system which continuously in the regular interval of time measuring the intensive parameter of the patient's health and if any abnormal condition occurs.

TABLE I LI-FI/WI-FI COMPARISON

\begin{tabular}{|l|c|c|}
\hline \multicolumn{1}{|c|}{ Parameter } & Li-Fi & Wi-Fi \\
\hline Speed & $\mathrm{H}$ & $\mathrm{H}$ \\
\hline Range & $\mathrm{L}$ & $\mathrm{M}$ \\
\hline Data density & $\mathrm{H}$ & $\mathrm{L}$ \\
\hline Security & $\mathrm{H}$ & $\mathrm{M}$ \\
\hline Reliability & $\mathrm{M}$ & $\mathrm{M}$ \\
\hline Power availability & $\mathrm{H}$ & $\mathrm{L}$ \\
\hline Transmit/receive power & $\mathrm{H}$ & $\mathrm{M}$ \\
\hline Ecological impact & $\mathrm{L}$ & $\mathrm{M}$ \\
\hline Device-to-device connectivity & $\mathrm{H}$ & $\mathrm{H}$ \\
\hline Obstacle interference & $\mathrm{H}$ & $\mathrm{L}$ \\
\hline Bill of materials & $\mathrm{H}$ & M \\
\hline Market maturity & $\mathrm{L}$ & $\mathrm{H}$ \\
\hline H: High, M: Medium, L: Low, Li-Fi: Light fidelity, Wi-Fi: Wireless fidelity \\
\hline
\end{tabular}

In this system the information about patient's health is provided within every prescribed interval of time to the Doctor. This data is provided to doctor via Wireless LI-FI modem. This system takes care of patient's health $24 \times 7$ whereas this facility is not available in the conventional system. This proposed system is portable \& can be used at the home also. Patient is connected with biomedical sensors, which captures physiological changes in real time and convert them into e-data, then compares it with predefined values or if any discrepancies are noticed, notification will be communicated to the doctors or emergency medical staff (nurses and technicians) on their handheld devices. Fig.1 shows the working method of Li-Fi technology.

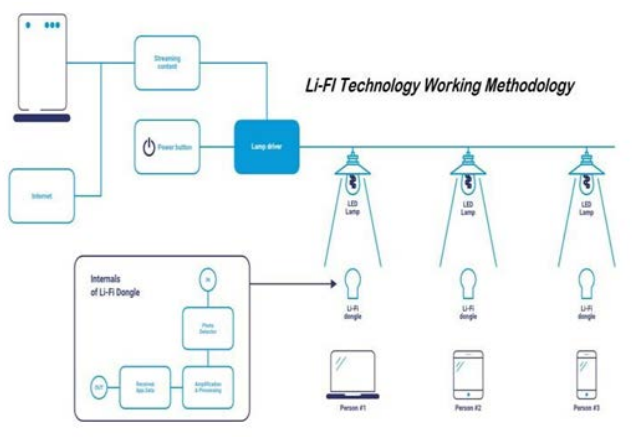

Fig. 1 Architecture

\section{APPLICATIONS ANDIMPLEMENTATION}

Apart from the usage in monitoring of patients two very important services are emergency alert Medical claim andRobotics surgery. Li-Fi is particularly suitable for many popular internet "content consumption” applications such as video and audio downloads, live streaming, etc. These applications place heavy demands on the downlink bandwidth but require minimal uplink capacity. In this way, the majority of the internet traffic is off-loaded from existing RF channels, thus also extending cellular and Wi-Fi capacities. Fig. 2 shows the applications of Li-Fi technology in hospitals.

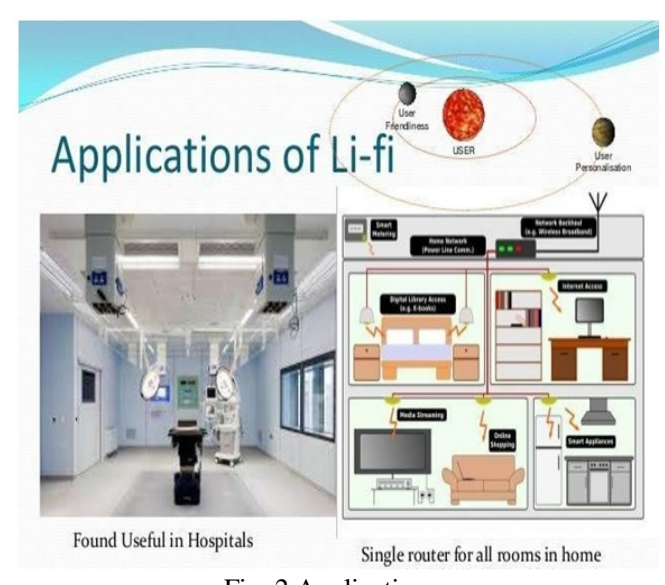

Fig. 2 Applications

\section{CONCLUSION}

The system was developed only with email provision but we have also implemented the SMS technique. In Fog Computing we presenting a new approach for solving the problem of insider data theft attacks in a cloud using dynamically generated decoy files and also saving storage required for maintaining decoy files in the cloud. Thus, it provides more security in cloud computing. 


\section{REFERENCES}

[1] MalekBenslama and HatemMokhtari, "Compressed Sensing in LIFI and WIFI networks”, Elsevier 2017 Edition

[2] Dean.A. Gratton, "Developing practical Wireless applications”, Elsevier, 2007.

[3] Akassh A. Mishra and Neelesh S. Salian, "Internet using Visible Light Communication”, IACSIT International Journal of Engineering and Technology, Vol. 3, No.5, October 2011

[4] T.Komine and M. Nakagawa , "Fundamental Analysis for VisibleLight Communication System Using LED Lights" , IEEETransactions on Consumer Electronics, Vol. 50, No.1, pp. 100107, February 2004

[5] Emilie bialic, Dinhchuong and David vaufrey, "Optimizing the rate in the LiFi System” US Patent9544053B2,May 2014
[6] [Online] Available: http:/www.lificonsortium.org

[7] G.Povey, Top 10 Li-Fi myths. Visible Light Commun.2012. [Online] Available: http://visiblelightcomm.com/top-10-li-fiMyths/Accessed on 22 June 2018.

[8] LIFI Technology for Indoor Access, Li-Fi, Mar2015

[9] Purelifi.com/wp-content/uploads/2017/12/LiFi-XC-Data-sheetSnapshot.pdf

[10] H. Elgala,"A Study on the Impact of Nonlinear Characteristics of LEDs on Optical OFDM," PhD Thesis, 2010.

[11] IvicaStevanovic , "OF COM report on Light fidelity”, Tech. Rep, May 2017

[12] Wireless LAN Medium Access Control (MAC) and Physical Layer (PHY) Specification, IEEE, Std. 802.11,1997. 